\title{
Feature Extraction for Iris Recognition Based on Optimized Convolution Kernels
}

\author{
Lubos Omelina $^{1,3}$, Bart Jansen ${ }^{1,2}$, Milos Oravec ${ }^{3}$, and Jan Cornelis ${ }^{1}$ \\ ${ }^{1}$ Department of Electronics and Informatics, Vrije Universiteit Brussel, Brussels, Belgium \\ ${ }^{2}$ iMinds, Dept. of Future Media and Imaging, Ghent, Belgium \\ ${ }^{3}$ Institute of Computer Science and Mathematics, \\ Slovak University of Technology in Bratislava, Slovakia \\ lomelina@etro.vub.ac.be, bjansen@etro.vub.ac.be, \\ milos.oravec@stuba.sk, jpcornel@etro.vub.ac.be
}

\begin{abstract}
Iris recognition has gained a lot of popularity for the last decades. Mainly a method based on binary iris templates found its way to real world use due to its simplicity, stability and reliability. The principle is that the unique iris structure is encoded to the bit code templates that are sufficient for high accuracy recognition. Encoding is performed by filtering a preprocessed iris image and storing only the phase information of the response to the filters. For years researchers used the 2D Gabor filters or their modifications, because these filters proved to provide the most reliable features. Despite the high recognition accuracy, the use of 2D Gabor filters faces a problem of spoofing. Recent studies show that the encoding process can be reverted and a spoofed iris can be obtained only based on the iris code. In this paper, we propose an efficient feature extraction method for iris recognition based on convolution kernels, learned from a database of irises. We show that the proposed method reaches state-of-the-art performance and can prohibit attackers from generating spoofed irises if the optimized convolution kernel is safely stored.
\end{abstract}

Keywords: iris recognition, simulated annealing, image filtering.

\section{Introduction}

An iris is a popular biometric trait used for recognizing humans. Its unique texture, stability over time, accessibility and the possibility of touchless scanning determine its suitability for biometric recognition. John Daugman proposed a method which reaches nearly $100 \%$ recognition accuracy even in large-scale scenarios with millions of subjects [1], [2]. His method uses 2D extensions of Gabor wavelets and their implementation through band-pass filters a.k.a. 2D Gabor filters [3]. They belong to the best features used for recognition tasks not only in biometrics. Recent research in iris recognition is focusing on methods for learning the optimal parameters of the Gabor kernels from limited data and on methods to minimize the number of features used in the recognition process. It was shown that 2D Gabor filters are an approximation of the visual system of mammals [3] and this is the main argument of many 
researchers for their use. It is not clear if the Gabor filters are the best filters for encoding the iris texture or even better solutions can be found. The visual system of mammals performs many other tasks besides recognition; so the similarity to it does not guarantee the best solution for recognition tasks. The popularity of Gabor features exceeds the iris recognition area and we believe that any improvement will hence also find its place in the broader image processing domain.

In this paper, we propose an efficient feature extraction method for iris recognition based on optimized convolution kernels. Rather than optimizing the parameters of a specific kernel type, we start from a randomly initialized convolution kernel and we optimize this kernel with the simulated annealing algorithm. This approach is not biased by any imposed mathematical properties of the kernel, at the price of a much larger search space. Our experiments show that the proposed method of feature extraction in iris recognition can reach comparable results to the state-of-the-art method proposed by J. Daugman and in some cases can even outperform his method. In addition, we found out that our approach can evolve an entire class of different convolution kernels, all resulting in very high iris recognition rates. This paper argues that the existence of such a class of (optimal) kernels can be a huge benefit for iris encoding and can prevent iris spoofing as there are recent studies that show the possibility of spoofing when Daugman's method based on 2D Gabor wavelets is used [4].

\subsection{A Short Review of Iris Recognition and Related Work}

Any image based biometric process begins with an image acquisition, its preprocessing and segmentation. In case of iris recognition, we need to localize the iris boundaries, eyelids and eyelashes. Then the image is segmented and the iris texture is transformed from Cartesian coordinates to the polar coordinate system. The circular band is divided into subbands of equal thickness. These subbands are averaged over a patch of pixels uniformly in radius r. From this unwrapped representation, features are extracted using Gabor filters and the result is encoded to a binary template (Figure 1). For matching two different irises the Hamming distance is used.

The most popular method for iris recognition, proposed by John Daugman [1], uses 2D Gabor wavelets for feature extraction. In iris recognition, 2D Gabor wavelets are implemented as a band-pass filter bank, because filters are more computationally efficient than computation of bi-orthogonal wavelets. Although variations of Daugman's method have been proposed, the most accurate ones are still based on the variations of Gabor filters [5] (e.g. log-Gabor filters [6, 7]).

The feature extraction from a preprocessed iris texture is done simply by filtering with 2D Gabor filters. The 2D Gabor filter can be understood as a Gaussian function modulated by a complex harmonic function:

$$
\begin{gathered}
G_{\left[x_{0}, y_{0}, \alpha, \beta, u_{0}, v_{0}\right]}(x, y)=\exp \left\{-\pi\left(\frac{\left(x-x_{0}\right)^{2}}{\alpha^{2}}+\frac{\left(y-y_{0}\right)^{2}}{\beta^{2}}\right)\right\} \\
\exp \left\{-2 \pi i\left(u_{0}\left(x-x_{0}\right)+v_{0}\left(y-y_{0}\right)\right\}\right.
\end{gathered}
$$

where $\left(x_{0}, y_{0}\right)$ specify position in the image, $(\alpha, \beta)$ specify the effective width and length, and $\left(u_{0}, v_{0}\right)$ specify modulation, which has spatial frequency $\omega_{0}=\sqrt{u_{0}^{2}+v_{0}^{2}}$ 
We consider that filtering is represented as a convolution in the image domain and thus from equation 1 we get two convolution kernels - one for the real part and a second one for the imaginary part. By convolving the iris image with the kernels we obtain responses to the 2D Gabor filters (a.k.a. Gabor features). Gabor features are encoded by quantizing the phase information into four levels for each quadrant in the complex plane. The phase information contains the most significant information [8] and is most relevant for the iris recognition. In terms of single response (either for the real or imaginary part) the pixel value in the response is encoded as 1 , if the value is positive and 0 otherwise.

a)

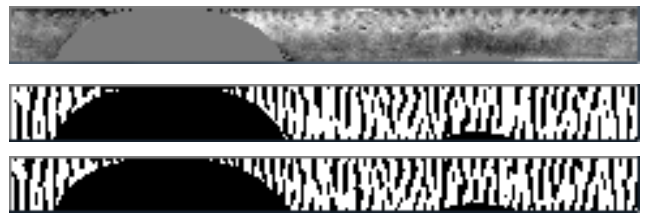

Fig. 1. An example of an unwrapped version of an iris and its corresponding iris code for the real (b) and the imaginary (c) part of the 2D Gabor filter

Despite the success of the 2D Gabor filters in the iris recognition, it is a complex task to design a good set of filters due to the large number of parameters. Tsai, Taur, $\&$ Tao proposed to use particle swarm optimization to estimate the best parameters of Gabor filters [9]. They improved the equal error rate on the CASIA-IrisV3-Interval database in comparison to Daugman's method and reduced the length of the template by $21.9 \%$. Z. Lin \& B. Lu [10] optimized multi-directional Gabor filters based on the knowledge from neurophysiological research to separate the filters for each sub-block in the effective iris area. H. Zheng and F. Su [11] used only the imaginary part of complex 2D Gabor filters to make the code shorter. They report no performance drop in the recognition accuracy.

\section{Method (Algorithm Overview)}

As we described in the previous section, the feature extraction from a preprocessed iris is done by filtering. In our work we assume that filtering is implemented through convolution and we intend to improve the recognition by replacing the Gabor kernel by a kernel optimized with the simulated annealing algorithm. Simulated annealing is a generic optimization algorithm locating the global optimum that works in a straightforward manner. We have chosen this algorithm mainly for the visibility of the optimization process to demonstrate the concept of optimizing the convolution kernel. However, other evolutionary algorithms can be applied to this problem as well and might reach even better results. We consider it to be a strength of our approach that even by using naïve optimization methods, we can in some cases outperform the Gabor based kernels. To be able to assess a single kernel, we simplified the feature extraction in the iris recognition method and used only a single convolution kernel for 
iris encoding; the rest of the recognition method remains the same as described in the previous section.

\subsection{Simulated Annealing Based Optimization of the Convolution Kernel}

Stochastic optimization and evolutionary algorithms are important optimization techniques, especially when no analytical solution is available. Simulated annealing [12] is a neighborhood search algorithm similar to simple local search, e.g., Hill Climbing, but with modified acceptance criteria permitting occasional worse solution during the computation. The main procedure is defined as follows: first, a stochastic solution is initialized randomly; then its neighbor, i.e. a new solution, is iteratively constructed. The occasional worse solution can save the algorithm from getting trapped in local optima. The simulated annealing algorithm hence requires the modeling of the problem in such a way that it is possible to move the partial solution from one state to another one (called a neighborhood state) which is generated randomly.

For the simulated annealing we must define several parameters. The first is an energy function $E(K)$ which evaluates the quality of the generated kernel solution $K$. In order to increase the overall recognition rate, we evaluate the recognition accuracy. The second parameter is the configuration space with the neighborhood function. In our case the configuration space is formed by the elements in the convolution kernel and the allowed intervals for these elements. The neighborhood function generates a new convolution kernel in each iteration. Third, the temperature and cooling schedule are adjusted empirically. In this work we explore 3 different energy functions:

Energy function 1: In this scenario we maximize the iris recognition accuracy $P(K)$. Thus, the energy function has only one component and is defined as follows:

$$
E_{1}(K)=P(K)
$$

Energy function 2: In the second scenario we also maximize the iris recognition accuracy, but once the accuracy reaches high values we continue the search process maximizing the distance between the genuine and the impostor distributions $D(K)$. We define the energy function as follows:

$$
E_{2}(K)=\left\{\begin{array}{lr}
P(K), & \text { if } P(K)<t \\
P(K)+D(K), & \text { otherwise }
\end{array}\right.
$$

where $P(K)$ is function providing iris recognition accuracy for the convolution kernel $K$ on the training set. $D(K)$ is defined as a distance between the genuine and the impostor distributions.

$$
D(K)=\left(\mu_{\text {impostor }}-\sigma_{\text {impostor }}\right)-\left(\mu_{\text {genuine }}+\sigma_{\text {genuine }}\right)
$$

where $\mu_{\text {impostor }}, \mu_{\text {genuine }}$ are means and $\sigma_{\text {genuine }}, \sigma_{\text {genuine }}$ are standard deviations of the distributions. 
Energy Function 3: In the third scenario we maximize the iris recognition accuracy and also the distance between the genuine and the impostor distributions at the same time. We define the energy function as a sum of both parts - the recognition accuracy and distance between the genuine and the impostor distributions:

$$
E_{3}(K)=P(K)+D(K)
$$

At each temperature, our algorithm generates a modified convolution kernel by choosing two random points in the kernel and adding a random value in the range $<-1,1\rangle$ to both of them. If the recognition accuracy increases over the previous convolution kernel, the new kernel is accepted in the $j$-th iteration (similar to the gradient method). Otherwise, when similarity is equal or decreases, a new kernel is accepted if

$$
e^{\frac{-\left(E\left(K_{j}\right)-E\left(K_{j-1}\right)\right)}{T_{j}}}>\xi
$$

where $\xi$ is randomly chosen from the interval $[0,1] \cdot E\left(K_{j}\right)$ is the energy of the convolution kernel generated in the $j$-th iteration. $T_{j}$ is the temperature in the $j$-th iteration. We use an exponential cooling scheme where $T_{j}=T_{j-1} \alpha \cdot \alpha$ is a constant defining the speed of cooling ( $\alpha=0.99$ ).

\subsection{Software Optimizations}

By the definition of our energy function, it is necessary to recompute the recognition accuracy at each iteration. By proposing two key optimizations we reduced the computation time from several months to days or even hours:

Optimized Hamming Distance Implementation. Computation of the Hamming distance is the most frequent operation required for computing the recognition accuracy. The distance is computed for each template comparison where each testing template is compared with all trained templates stored in the database. Inputs for the Hamming distance operation are 2 iris templates. The computation consists of two main operations:

- $\quad$ Exclusive-OR (XOR) to check which bits are different in the templates.

- $\quad$ Sum of ones in the result of the XOR.

The result of the convolution is a matrix containing floating point values. These values are then encoded to zeros and ones. A natural and faster way of encoding is to modify this matrix directly to contain only zeros and ones. We decided to encode each column (containing 20 values in our case) of the matrix to a single 32 bit value, allowing us to compare two template columns with a single XOR instruction. Using SSE instructions, 4 columns can be processed simultaneously. 
Iterative Convolution Computation. Since we defined the neighborhood functions as a limited change of the selected elements in the convolution kernel, it is necessary to also recompute the iris codes for all the irises in the database. By using dynamic programming principles we can reduce the computation time of the convolution in following way: Let $f$ be an iris image and $g$ the convolution kernel generated in the iteration. Then the convolution is defined as follows:

$$
\left(f * g_{i}\right)[n]=\sum_{m=0}^{N-1} f[n-m] g_{i}[m]
$$

If we define the neighborhood function as a change of a single element $k$ in the convolution kernel by value $\delta$ then

$$
g_{i+1}[k]=g_{i}[k]+\delta
$$

and

$$
\left(f * g_{i+1}\right)[n]=\sum_{m=0}^{N-1} f[n-m] g_{i}[m]+f[n-k] \delta=\left(f * g_{i}\right)[n]+f[n-k] \delta
$$

where $\left(f * g_{i}\right)[n]$ is the convolution from the iteration $i$. This means that we can reduce the summation over $\mathrm{N}$ elements to the summation over two elements if we use the result of the convolution from the previous iteration. In case of a squared kernel containing 14 14=196 elements, we can avoid 194 of the 195 matrix additions.

\section{Experimental Results}

The proposed algorithm was tested on the Casia v1.0 [13] iris database. This database contains 756 grayscale images of 108 irises. The size of these images is $320 \times 240$ pixels. All images from the database were automatically preprocessed and segmented using existing software packages [6]. The preprocessing of an iris is not an easy task and can fail for a variety of reasons. The rate, at which creating a template from an input is unsuccessful, is called failure to enroll rate (FTE). This is usually caused by low quality input images. The rate at which the system fails to detect an iris when the image is correctly presented is called failure to capture rate (FTC). Although the CASIA v1.0 database contains 756 images, only 631 images were successfully preprocessed by the automatic preprocessing algorithm. In all our experiments we use only the 631 correctly enrolled images.

We split our database in two parts, each containing 54 irises and 315.5 images on average. We use the first half for learning the kernel. The second half is used for testing purposes. It is important to note that the training and the testing set should not contain images of the same irises even if these images are different. We do the repeated random sub-sampling cross-validation, i.e. we repeat experiments 10 times to obtain meaningful results that are not based on a specific set of images. The average recognition accuracy of the 10 repetitions for each energy function is listed in the Table 1. 
Table 1. Recognition accuracy computed for different energy functions

\begin{tabular}{|l|c|c|c|c|}
\hline & Best Gabor & \multicolumn{3}{|c|}{ Optimized kernels } \\
\cline { 3 - 5 } & kernel & E 1 & E2 & E3 \\
\hline Recognition & 99,058 & 98,798 & 99,922 & 99,57 \\
Accuracy $(\%) *$ & & $( \pm 0,645)$ & $( \pm 0,158)$ & $( \pm 0,404)$ \\
\hline
\end{tabular}

* Results for optimized kernels are average of 10 experiments.

The only common parameter between the Gabor kernel and the randomly generated kernel is the kernel size. In our experiments we use a size of 14 pixels. For this kernel size the Gabor kernel performs best.

We analyzed the influence of the size and the modulation frequency of the 2D Gabor filter on the recognition accuracy and compared our results against the best performing kernels we could obtain. The highest recognition accuracy (99,058\%) was observed for more parameter combinations (e.g. kernel size of $7 \mathrm{px}$ and frequency 1.1). The search for the right size of the Gabor kernel is important mainly because it depends on the resolution of the preprocessed iris image, thus can vary depending on the preprocessing.

We evaluated the proposed feature extraction method for all 3 energy functions (Table 1) described in the previous section. The recognition accuracy we reached with a single convolution kernel is $98.798,99.922 \%, 99.57 \%$ respectively for the energy functions E1, E2 and E3. For energy function E2, in 5 out of the 10 experiments we reached $100 \%$ recognition accuracy on the test set and we reached $100 \%$ recognition accuracy on the training set in all cases for E2. For the three studied energy functions, the results are not significantly different from the best Gabor kernel we could find (99.058\% recognition accuracy).

In the Figure 3 examples of optimized convolution kernels are shown. Initial results seem to indicate that using E1, no pattern can be visually detected in the optimized kernels, while kernels resulting from E2 and E3 show some structure. Further analysis on the interpretation of these observed differences is required.

Optimization with the energy function E1 needs 2911 iterations to reach the maximal energy (which is equal to $100 \%$ recognition accuracy on the training set). We did not specify maximal energy in experiments with energy functions E2 and E3 - the experiments were limited by the maximal number of iterations which is defined by parameters of the simulated annealing algorithm (138149 iterations in our case).

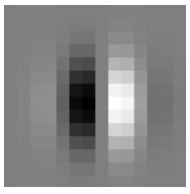

a)

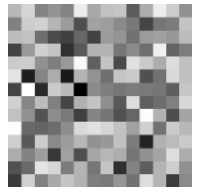

b)

Fig. 2. Convolution kernels used for feature extraction. a) Imaginary part of 2D Gabor filter reached 99,058 \% recognition accuracy. b) An example of the optimized kernel which reached $100 \%$ recognition accuracy. 

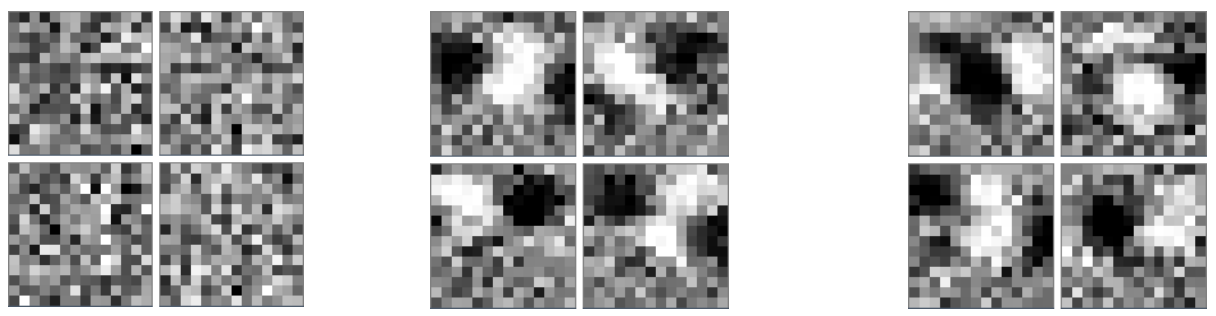

Fig. 3. Visualization of 4 different optimized convolution kernels for each energy function, on the left - E1, in the center - E2, on the right - E3. All of these convolution kernels reached more than $97 \%$ recognition accuracy.

This study demonstrates the real potential of learning convolution kernels in a scenario where only a single convolution kernel is used. We believe that there is also potential of using multiple convolution kernels as it is used in other iris recognition methods. The use of multiple kernels allows nonlinear higher order generalizations of convolution classifiers. Nonlinear convolution classifiers can lead to stronger aggregated classifiers [14].

\section{$4 \quad$ Security Aspects}

It was shown by John Daugman that iris templates are unique across people [1] and it was generally accepted that the templates are representing unidentifiable data. Recent studies show that this assumption is not correct and that it is possible to generate spoofed irises from an iris code template [4, 15]. Venugopalan and Savvides presented a method for obtaining spoof irises by synthetizing several levels of natural looking irises [4]. Their approach assumes that the feature extraction mechanism is exposed and uses the Gabor kernel. They advise to secure the database with iris templates in order to be sure no attacker can access them.

Our experiments demonstrate the existence of a whole class of convolution kernels that can reach the same results as 2D Gabor filters when used for iris encoding (See figure 3 for some examples). The result of each optimization is a convolution kernel with unknown analytical definition and pseudo-random values. In contrast to traditional feature extraction, in our approach we use these pseudo-random kernels (obtained by optimizing randomly initialized kernels) that lead to different iris codes (see Figure 4). By filtering an iris image with 2D Gabor filters - which are also used as edge detector - and encoding it, we obtain a code that contains visible structure (see Figure 4a). Since the template is an already encoded iris, it contains the most important information from the iris structure that can be expressed in terms of 2D Gabor filters as an edge with specific response to the filters. Thanks to the analytical definition of 2D Gabor wavelets we can revert the operation and get an approximation of an iris generating the same code [4]. On the other hand, the code generated from evolved pseudo-random kernels does not contain a visible structure in form of edges (see Figure 4b). If we assume that our optimized convolution kernel is safely stored within a biometric system, the filtering process cannot easily be reverted for the generation of a spoofed iris texture. 
a)

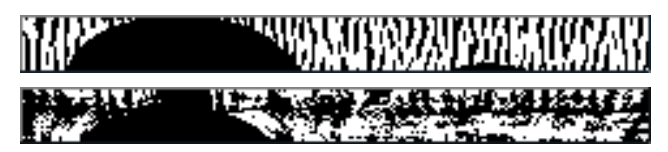

Fig. 4. Two iris codes generated for the same iris image, (a) an iris code generated with imaginary part of the 2D Gabor filter, (b) an iris code generated with randomly initialized and optimized convolution kernel

\section{Conclusion}

In this paper, we propose a novel feature extraction method for iris recognition based on binary templates. We improve the feature extraction by replacing the $2 \mathrm{D}$ Gabor kernel by a kernel optimized with the simulated annealing algorithm. The algorithm is simple and can find a global solution (if it exists). Our experiments with simulated annealing show that the proposed feature extraction method can reach comparable results to the state-of-the-art method [1]. In addition, our experiments reveal that there is a class of convolution kernels suitable for feature extraction from the iris texture that can perform at least as good as 2D Gabor kernels when a single convolution kernel is used. Systems based on our approach are more difficult to spoof, as the current spoofing techniques require the use of Gabor features. To the best of our knowledge, no similar work currently exists that uses a stochastic evolution algorithm to optimize convolution kernels for the iris feature extraction. As future work, we will verify our results on different larger iris databases.

Acknowledgments. Research described in the paper was done within the grants No. 1/0529/13, 1/0961/11 of the Slovak Grant Agency VEGA, HBBNext FP7-ICT-20117-287848 project and within the ICT4Rehab project (www.ict4rehab.org) funded by Innoviris. Portions of the research in this paper use the CASIA-V1 collected by the Chinese Academy of Sciences' Institute of Automation (CASIA).

\section{References}

1. Daugman, J.: How Iris Recognition Works. IEEE Transactions on Circuits and Systems for Video Technology 14, 21-30 (2004)

2. Daugman, J.: The importance of being random: statistical principles of iris recognition. Pattern Recognition 36, 279-291 (2003)

3. Daugman, J.: Uncertainty relation for resolution in space, spatial frequency, and orientation optimized by two-dimensional visual cortical filters. Journal of the Optical Society of America A: Optics, Image Science, and Vision 2, 1160-1169 (1985)

4. Venugopalan, S., Savvides, M.: How to Generate Spoofed Irises From an Iris Code Template. IEEE Transactions on Information Forensics and Security 6, 385-395 (2011)

5. Bowyer, K.W., Hollingsworth, K., Flynn, P.J.: Image understanding for iris biometrics: A survey. Computer Vision and Image Understanding 110, 281-307 (2008)

6. Masek, L.: Recognition of Human Iris Patterns for Biometric Identification (2003),

http: //citeseerx.ist.psu.edu/viewdoc/

summary?doi=10.1.1.90.5112 
7. Yao, P., Li, J., Ye, X., Zhuang, Z., Li, B.: Iris Recognition Algorithm Using Modified Log-Gabor Filters. In: 18th International Conference on Pattern Recognition (ICPR 2006), pp. 461-464. IEEE (2006)

8. Oppenheim, A.V., Lim, J.S.: The importance of phase in signals. Proceedings of the IEEE 69, 529-541 (1981)

9. Tsai, C.C., Taur, J.S., Tao, C.W.: Iris recognition using Gabor filters optimized by the particle swarm technique. In: 2008 IEEE International Conference on Systems, Man and Cybernetics, pp. 921-926. IEEE (2008)

10. Lin, Z., Lu, B.: Iris recognition method based on the optimized Gabor filters. In: 2010 3rd International Congress on Image and Signal Processing, pp. 1868-1872 (2010)

11. Zheng, H., Su, F.: An improved iris recognition system based on gabor filters. In: IEEE International Conference on Network Infrastructure and Digital Content, IC-NIDC 2009, Beijing, China, pp. 823-827 (2009)

12. Kirkpatrick, S., Gelatt Jr., C.D., Vecchi, M.P.: Optimization by simulated annealing. In: Readings in Computer Vision: Issues, Problems, Principles, and Paradigms, pp. 606-615. Morgan Kaufmann Publishers Inc., San Francisco (1987)

13. CASIA Iris Image Database, N. L. of Pattern Recognition (NLPR), Institute of Automation (AI) Chinese Academy of Science, http : / / biometrics. ideal test.org

14. Kumar, R., Banerjee, A., Vemuri, B.C., Pfister, H.: Trainable Convolution Filters and their Application to Face Recognition. IEEE Transactions on Pattern Analysis and Machine Intelligence 34 (2011)

15. Galbally, J.: From the iriscode to the iris: a new vulnerability of iris recognition systems. Black Hat USA 2012 (2012) 